\title{
New Interpretations of Normalization Methods in Deep Learning
}

\author{
Jiacheng Sun, ${ }^{1}$ Xiangyong Cao, ${ }^{2}$ Hanwen Liang, ${ }^{1}$ Weiran Huang, ${ }^{1}$ Zewei Chen, ${ }^{1}$ Zhenguo Li ${ }^{1}$ \\ ${ }^{1}$ Huawei Noah's Ark Lab, ${ }^{2}$ Xi' an Jiaotong University \\ \{sunjiacheng1, lianghanwen1, weiran.huang, chen.zewei, Li.Zhenguo\}@ huawei.com, caoxiangyong@ mail.xjtu.edu.cn
}

\begin{abstract}
In recent years, a variety of normalization methods have been proposed to help training neural networks, such as batch normalization $(\mathrm{BN})$, layer normalization (LN), weight normalization (WN), group normalization (GN), etc. However, some necessary tools to analyze all these normalization methods are lacking. In this paper, we first propose a lemma to define some necessary tools. Then, we use these tools to make a deep analysis on popular normalization methods and obtain the following conclusions: 1) Most of the normalization methods can be interpreted in a unified framework, namely normalizing pre-activations or weights onto a sphere; 2) Since most of the existing normalization methods are scaling invariant, we can conduct optimization on a sphere with scaling symmetry removed, which can help to stabilize the training of network; 3) We prove that training with these normalization methods can make the norm of weights increase, which could cause adversarial vulnerability as it amplifies the attack. Finally, a series of experiments are conducted to verify these claims.
\end{abstract}

\section{Introduction}

Normalization has been a very effective strategy in deep learning, which speeds up training deep neural networks and also acts as a regularizer to improve generalization. This technique has been a fundamental component in many stateof-the-art algorithms, and is usually implemented by adding non-linear mappings to pre-activations or weights before activation functions. In recent years, a variety of normalization methods have been proposed, including batch normalization (BN) (Ioffe and Szegedy 2015), layer normalization (LN) (Ba, Kiros, and Hinton 2016), instance normalization (IN) (Ulyanov, Vedaldi, and Lempitsky 2016), group normalization (GN) (Wu and He 2018), weight normalization (WN) (Salimans and Kingma 2016), centered weight normalization (CWN) (Huang et al. 2017), spectral normalization (SN) (Miyato et al. 2018), etc. These methods can be roughly divided into two categories: data-based normalization method and weight-based normalization method. In the following, we will first review the two categories, and then introduce the motivation and contribution of our work.

Copyright (c) 2020, Association for the Advancement of Artificial Intelligence (www.aaai.org). All rights reserved.

\subsection{Related work: data-based normalization method vs weight-based normalization method}

Data-based normalization method is implemented by normalizing the pre-activations/activations (Mishkin and Matas 2015), which is computed by passing the data into each layer of the network. The typical methods include BN, LN, IN and GN. More specifically, BN standardizes the pre-activation vector by transforming it into a vector with zero mean and unit variance. $\mathrm{LN}$ implements this standardization within one layer, and this method is equivalent to first centering every column of weights and then conducting the multiplicity and scaling. Therefore, it is scaling invariant with both weights and data as well as moving invariant in a certain direction. IN implements the standardization in one channel of the pre-activation, while GN balances between $\mathrm{LN}$ and IN and is implemented by dividing the channels into groups.

Comparatively, the other strategy weight-based normalization method normalizes the weights directly. The typical methods are WN, CWN and SN. Specifically, WN (Salimans and Kingma 2016) decouples the length and direction of the weight vector as BN does. CWN (Huang et al. 2017) standardizes the weight vector by centering at mean and scaling by its $L_{2}$ norm and is equivalent to weight standardization (WS) (Qiao et al. 2019). SN is another normalization method that helps to stabilize the training of discriminator in the generative adversarial network (GAN), and it is similar to WN in dividing the spectral norm of the weight matrix. This method is implemented by controlling the Lipschitz constant not too big, so it can be much more robust to noise (Miyato et al. 2018).

\subsection{Motivations}

Although various normalization methods have been proposed and verified to be effective in many computer vision tasks, there still exist many issues. Firstly, all the existing normalization methods are heuristic and lack of a unified theoretical understanding. Therefore, it is very important to interpret all the normalization methods from a theoretical perspective. Secondly, many normalization methods are widely used and their performance is closely related to the batch size. Take BN for instance. The small batch size makes the network training rather unstable ( $\mathrm{Wu}$ and $\mathrm{He}$ 
2018), while a large batch size may decrease the generalization capacity (Luo et al. 2019). Therefore, it is quite the fundamental problem to choose the optimal batch size for different tasks in deep learning (Masters and Luschi 2018; Park et al. 2019). Besides, the scaling parameters $\gamma$ of $\mathrm{BN}$ have been adopted as criteria for pruning network (Liu et al. 2017), which is implemented by removing units whose scaling parameter $\gamma$ is small. This indicates that the parameters $\gamma$ in $\mathrm{BN}$ are very important in evaluating a channel/neuron. In this work, we denote that $\gamma$ and $\beta$ are the approximation of standard deviation and mean in a batch. If the $\gamma$ of a channel is small, the output of this channel is approximated by a constant and contains little information for the next layer. Thus, it is reasonable to prune channels with small $\gamma$ as (Liu et al. 2017) does. Thirdly, the scaling invariant property and symmetry of normalization methods need to be carefully studied since it can influence the stability of training the network.

\subsection{Contributions}

To tackle the aforementioned issues, we propose a corresponding scheme. More specifically, our contributions are three-fold: (1) we first propose a unified analysis tool for most of the existing normalization methods. Then, we make a deep analysis of normalization methods according to the proposed tools and point out the relationship between them; (2) we put forward some useful claims and conclusions. First, almost all the normalization methods can be interpreted as normalizing pre-activations or weights onto a sphere or ellipsoid; Second, conducting optimization on a sphere with scaling symmetry removed could help to make the training of network more stable; (3) we show that training with these normalization methods will keep weights increasing, which will cause adversarial vulnerability since it will amplify the attack.

The rest of the paper is organized as follows: In Section 2 , we interpret different normalization methods in a unified analysis framework and propose some useful claims and conclusions. We conduct several experiments to verify these claims in Section 3 and conclude in Section 4.

\section{Interpretations of Normalization Methods}

As mentioned in the introduction section, a variety of normalization methods have been proposed in recent years (Ioffe and Szegedy 2015; Ulyanov, Vedaldi, and Lempitsky 2016; Wu and He 2018; Salimans and Kingma 2016; Huang et al. 2017; Miyato et al. 2018). In this section, we first give a unified analysis tool by proposing a lemma to exhibit the basic structure underlying these methods. Then, we make a deep analysis of each normalization method and put forward some useful claims and conclusions.

\subsection{A Unified Analysis Tool}

Before analyzing each normalization method, we first propose a lemma to define the related notations used throughout the paper.

Lemma 1 Given a vector $v=\left(v_{1}, v_{2}, \ldots, v_{n}\right)^{T} \in \mathbb{R}^{n}$, then 1) its mean vector $\bar{v}=\frac{1}{n} e_{n}^{T} v e_{n}=\frac{1}{n} e_{n} e_{n}^{T} v$, where direction $e_{n}=(1,1, \ldots, 1)^{T} \in \mathbb{R}^{n}$;
2) the centered vector $v-\bar{v}=P_{e_{n}} v$, where $P_{e_{n}}=I_{n}-$ $\frac{1}{n} e_{n} e_{n}^{T}$ is the projection to the hyperplane with direction $e_{n}$. $P_{e_{n}}$ has some properties, such as $P_{e_{n}}^{2}=P_{e_{n}}$, and $P_{e_{n}} v \perp$ $e_{n}$

3) its variance $\sigma_{v}^{2}=\frac{1}{n}(v-\bar{v})^{T}(v-\bar{v})=\frac{1}{n}\|v-\bar{v}\|_{2}^{2}=$ $\frac{1}{n} v^{T} P_{e_{n}} v$, thus $\sigma_{v}=\frac{1}{\sqrt{n}}\|v-\bar{v}\|_{2}$;

4) the standardization

$$
N(v)=\frac{v-\bar{v}}{\sigma_{v}}=\sqrt{n} \frac{v-\bar{v}}{\|v-\bar{v}\|_{2}}=\frac{\sqrt{n}}{\left\|P_{e_{n}} v\right\|_{2}} P_{e_{n}} v,
$$

and the norm is

$$
\|N(v)\|_{2}=\sqrt{n}
$$

i.e. $N(v) \in \mathbb{S}_{\sqrt{n}}^{n-2}$, where $\mathbb{S}_{\sqrt{n}}^{n-2}$ represents the $n-2$ dimension sphere with radius $\sqrt{n}$ and center 0 (omitted when center at origin); Also, $N(v)$ is scaling and moving invariant, i.e. $N\left(\alpha v+t e_{n}\right)=N(v), \forall \alpha, t \in \mathbb{R}$;

$5)$ we have the orthogonal decomposition

$$
v=\gamma N(v)+\beta e_{n},
$$

where $\gamma=\sigma_{v}=\frac{\|v-\bar{v}\|_{2}}{\sqrt{n}}, \beta=\frac{1}{n} e_{n}^{T} v$. The norm is

$$
\begin{aligned}
\|v\|_{2}^{2}=\|v-\bar{v}\|_{2}^{2}+\|\bar{v}\|_{2}^{2} & =\gamma^{2}\|N(v)\|_{2}^{2}+n \beta^{2} \\
& =n\left(\gamma^{2}+\beta^{2}\right) .
\end{aligned}
$$

If $\gamma$ and $\beta$ are fixed, $\gamma N(v)+\beta e_{n} \in \mathbb{S}_{\gamma \sqrt{n}}^{n-2}\left(\beta e_{n}\right)$.

Proof 1 1) The mean of $n$-dimension vector $v \in \mathbb{R}^{n}$ is

$$
\begin{aligned}
& \frac{1}{n}\left(v_{1}+v_{2}+\ldots+v_{n}\right) \\
& =\frac{1}{n}(1,1, \ldots, 1) \cdot\left(v_{1}, \ldots, v_{n}\right)^{T} \\
& =\frac{1}{n} e_{n}^{T} v \in \mathbb{R}
\end{aligned}
$$

Note that the last equality holds as the multiplicity of scalar and vector can be commutative. Thus the mean vector

$$
\begin{aligned}
\bar{v} & =\frac{1}{n}\left(v_{1}+v_{2}+\ldots+v_{n}\right) e_{n} \\
& =\frac{1}{n}\left(e_{n}^{T} v\right) e_{n}=\frac{1}{n} e_{n}\left(e_{n}^{T} v\right)=\frac{1}{n} e_{n} e_{n}^{T} v
\end{aligned}
$$

2) By (6), the centered vector

$$
v-\bar{v}=v-\frac{1}{n} e_{n} e_{n}^{T} v=\left(I_{n}-\frac{1}{n} e_{n} e_{n}^{T}\right) v=P_{e_{n}} v
$$

Now we prove $P_{e_{n}}$ is a projection matrix, i.e., $P_{e_{n}}^{2}=P_{e_{n}}$.

$$
\begin{aligned}
P_{e_{n}}^{2} & =\left(I_{n}-\frac{1}{n} e_{n} e_{n}^{T}\right)\left(I_{n}-\frac{1}{n} e_{n} e_{n}^{T}\right) \\
& =I_{n}-\frac{2}{n} e_{n} e_{n}^{T}+\frac{1}{n^{2}} e_{n}\left(e_{n}^{T} e_{n}\right) e_{n}^{T} \\
& =I_{n}-\frac{2}{n} e_{n} e_{n}^{T}+\frac{1}{n} e_{n} e_{n}^{T} \\
& =I_{n}-\frac{1}{n} e_{n} e_{n}^{T}=P_{e_{n}}
\end{aligned}
$$




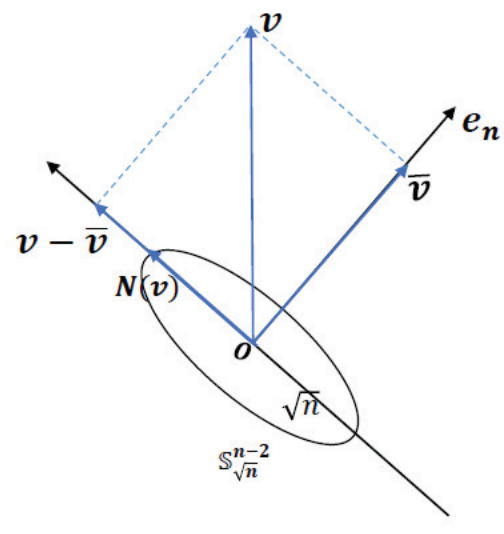

(a)

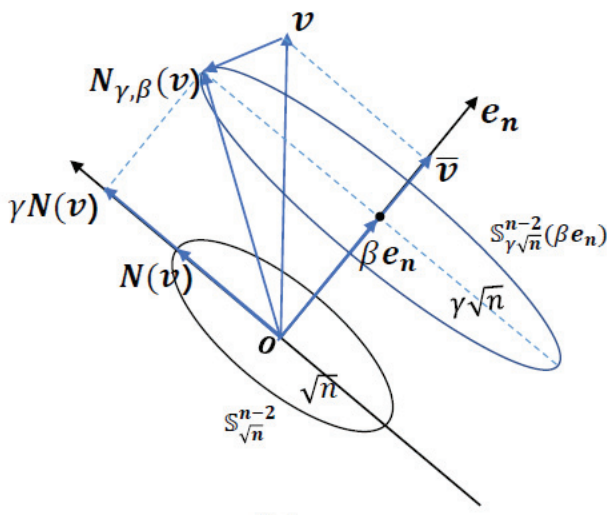

(b)

Figure 1: (a) The mean vector $\bar{v}$ of $v$ is its projection on $e_{n} \cdot v-\bar{v}$ is orthogonal to $e_{n}$ and parallel to $N(v) \in \mathbb{S}_{\sqrt{n}}^{n-2} \cdot(\mathrm{b})$ Given $\gamma$ and $\beta, N_{\gamma, \beta}(v)$ maps each $v$ onto $\mathbb{S}_{\gamma \sqrt{n}}^{n-2}\left(\beta e_{n}\right)$, which implies that vector $N_{\gamma, \beta}(v)$ lies on a sphere with radius $\gamma \sqrt{n}$ and center $\beta e_{n}$.

Note that the last equality holds as $e_{n}^{T} e_{n}=n$ by the definition of $e_{n}$.

Also we have

$$
\begin{aligned}
e_{n}^{T} P_{e_{n}} v & =e_{n}^{T}\left(I_{n}-\frac{1}{n} e_{n} e_{n}^{T}\right) v \\
& =\left(e_{n}^{T}-\frac{1}{n}\left(e_{n}^{T} e_{n}\right) e_{n}^{T}\right) v \\
& =\left(e_{n}^{T}-e_{n}^{T}\right) v=0
\end{aligned}
$$

So $P_{e_{n}} v \perp e_{n}$, and $P_{e_{n}}$ is the projection operation onto $e_{n}^{\perp}$. 3) The variance of components in $v$ is

$$
\begin{aligned}
\sigma_{v}^{2} & =\frac{1}{n} \sum_{i=1}^{n}\left(v_{i}-\frac{1}{n} e_{n}^{T} v\right) \\
& =\frac{1}{n}(v-\bar{v})^{T}(v-\bar{v})=\frac{1}{n}\|v-\bar{v}\|_{2}^{2} \\
& =\frac{1}{n}\left\|P_{e_{n}} v\right\|_{2}^{2}
\end{aligned}
$$

Thus, $\sigma_{v}=\frac{1}{\sqrt{n}}\|v-\bar{v}\|_{2}=\frac{1}{n}\left\|P_{e_{n}} v\right\|_{2}$.

4) We only need to show that $N(v)$ is scaling and moving invariant by above results. $\forall \alpha, t \in \mathbb{R}$,

$$
\begin{aligned}
& N\left(\alpha v+t e_{n}\right) \\
& =\sqrt{n} \frac{\left.\left(\alpha v+t e_{n}\right)-(\alpha \bar{v})+t e_{n}\right)}{\left.\|\left(\alpha v+t e_{n}\right)-(\alpha \bar{v})+t e_{n}\right) \|_{2}} \\
& =\sqrt{n} \frac{\alpha v-\alpha \bar{v}}{\alpha v-\alpha \bar{v}}=N(v)
\end{aligned}
$$

The norm of $N(v)=\sqrt{n}$ by definition, which means that $N(v)$ always lies on a sphere with radius $\sqrt{n}$ and center 0 which is orthogonal to fixed direction $e_{n}$. Thus $N(v) \in$ $\mathbb{S}_{\sqrt{n}}^{n-2}$
5) Now we prove the orthogonal decomposition of (3).

$$
\begin{aligned}
v & =v-\bar{v}+\bar{v} \\
& =\sigma_{v} \frac{v-\bar{v}}{\sigma_{v}}+\frac{1}{n} e_{n}^{T} v e_{n} \\
& =\sigma_{v} N(v)+\frac{1}{n} e_{n}^{T} v e_{n} \\
& =\gamma N(v)+\beta e_{n}
\end{aligned}
$$

where $\gamma=\sigma_{v}=\frac{\|v-\bar{v}\|_{2}}{\sqrt{n}}, \beta=\frac{1}{n} e_{n}^{T} v$.

Also from 2) we learn that $N(v) \perp e_{n}$, thus we have

$$
\begin{aligned}
\|v\|_{2}^{2} & =\|v-\bar{v}\|_{2}^{2}+\|\bar{v}\|_{2}^{2} \\
& =\gamma^{2}\|N(v)\|_{2}^{2}+n \beta^{2} \\
& =n\left(\gamma^{2}+\beta^{2}\right)
\end{aligned}
$$

Then for any vector we can composite it as two orthogonal direction $N(v)$ and $e_{n}$. If we fix the coefficients $\gamma, \beta$ by the definition of $N(v), \gamma N(v)+\beta e_{n} \in \mathbb{S}_{\gamma \sqrt{n}}^{n-2}\left(\beta e_{n}\right)$.

To better illustrate this lemma, a sketch map is shown in Fig. 1. To sum up, this lemma gives us an overall picture for understanding normalization. Most of the normalization methods standardize data or weights. More specifically, the main idea is to decouple the length and direction and optimize them separably (Kohler et al. 2019). Additionally, it can also be seen from the lemma that the standardized vectors lie on a sphere, and all directions are equilibrium on the sphere. Therefore, we can do optimization on a compact sphere instead of the whole parameter space. In the following, we will use this lemma to make a deep interpretation for some of the normalization methods.

\subsection{Batch Normalization}

In this section, we make a deep analysis of batch normalization $(\mathrm{BN})$ based on Lemma 1. For simplicity, we only an- 
alyze one layer in a multi-layer perceptron (MLP), while it is similar to analyze the convolution layer of CNN (more details can be seen in the Appendix of the supplemental material). There are also extensively works on batch normalization, for example, why it works (Yang et al. 2019; Cho and Lee 2017; Bjorck et al. 2018; Kohler et al. 2019) and how to use it in concrete tasks (Park et al. 2019), while we focus on its geometric properties.

Before conducting the analysis, some notations are defined. $\left\{X^{k}\right\}_{k=1}^{B}, X^{k} \in \mathbb{R}^{n}, k=1, \ldots, B$, denotes a batch set with $B$ samples, and the input for one layer can be represented as $X=\left(X^{1}, \ldots, X^{B}\right) \in \mathbb{R}^{n \times B}$. We assume that the layer of MLP contains $m$ hidden nodes, and thereby the weight matrix of this layer can be denoted as $W=\left(W_{1}, W_{2}, \ldots, W_{m}\right)^{T} \in \mathbb{R}^{m \times n}$, where each row vector $W_{i} \in \mathbb{R}^{n}, i=1, \ldots, m$. Therefore, the output $Y^{k}=$ $\left(Y_{1}^{k}, Y_{2}^{k}, \ldots, Y_{m}^{k}\right) \in \mathbb{R}^{m}$ of this layer can be represented as $Y^{k}=W X^{k}$. Additionally, we also define an all-one vector $e_{B}=(1,1, \ldots, 1)^{T} \in \mathbb{R}^{B}$.

Based on Lemma 1, batch normalization for $Y_{i}^{k}$ can be expressed as

$$
\begin{aligned}
& B N_{\gamma, \beta}\left(Y_{i}^{k}\right) \\
& =\gamma \sqrt{B} \frac{Y_{i}^{k}-\bar{Y}_{i}}{\left\|Y_{i}^{k}-\bar{Y}_{i}\right\|_{2}}+\beta e_{B}^{T} \in \mathbb{S}_{\gamma \sqrt{B}}^{B-2}\left(\beta e_{B}^{T}\right)
\end{aligned}
$$

where $Y_{i}^{k}$ is the $i_{t h}$ element of pre-activation vector $Y^{k}$, and $\bar{Y}_{i}$ is the mean of components for $Y_{i}^{k}$. Besides, $\left\|B N_{\gamma, \beta}\left(Y_{i}\right)\right\|_{2}=\sqrt{B\left(\gamma^{2}+\beta^{2}\right)}$. According to this new expression of $\mathrm{BN}$, we make several discoveries.

Firstly, since $B N_{\gamma, \beta}\left(Y_{i}\right) \in \mathbb{S}_{\gamma \sqrt{B}}^{B-2}\left(\beta e_{B}^{T}\right)$, training with $\mathrm{BN}$ can be interpreted as finding a fixed sphere, where the pre-activation vector $Y_{i}^{k}$ can be approximated well.

Secondly, the parameters $\gamma$ and $\beta$ are very important as they determine the sphere for pre-activation vectors. The only difference between units at training is the two parameters, and they can be treated as the characteristics of units. More concretely, units with similar $\gamma$ and $\beta$ have similar outputs and thus similar functions. Therefore, the performance of the network can still be maintained by removing some of the similar units. A similar idea has been applied to network pruning (Liu et al. 2017) by removing some units with small $\gamma$. But in our view, this idea can be further relaxed based on our observation. Specifically, this idea can be expressed as that not only units with small $\gamma$, but also units with similar $\gamma$ and $\beta$ can be removed. We will leave this method as a future work.

Thirdly, it also should be noted that the order of data in a batch does not change the result, thus the direction $N(v)$ can be any permutation of the components. There are $B$ ! possible directions in a batch in total, and these directions should have the same output.

Fourthly, batch size is a very important hyperparameter for training with BN. That too small batch size will not make the training stable and large batch size would decrease generalization as studied in (Luo et al. 2019). Batch normaliza- tion for $Y_{i}$ can be represented as

$$
B N\left(Y_{i}\right)=\frac{W_{i}(X-\bar{X})}{\sqrt{\frac{1}{B}\left\|W_{i}(X-\bar{X})\right\|_{2}^{2}}}=\frac{W_{i}(X-\bar{X})}{\sqrt{W_{i} \Sigma_{X} W_{i}^{T}}}
$$

where each row of $\bar{X}$ is the mean of row in $X, \bar{X}=$ $\frac{1}{B} X e_{B} e_{B}^{T}, \Sigma_{X}$ is the covariance matrix of row vectors in $X$, and $\Sigma_{X}=\frac{1}{B}(X-\bar{X})(X-\bar{X})^{T}$. We denote the rank of $\Sigma_{X}$ as $\operatorname{rank}\left(\Sigma_{X}\right)$, and $\operatorname{rank}\left(\Sigma_{X}\right) \leq \min (n, B)$. Next, we will have a further study about this by rank analysis.

- Let $K_{\Sigma_{X}}=\left\{W_{i} \in \mathbb{R}^{n} \mid W_{i} \Sigma_{X}=0\right\}$ be the kernel space of $\Sigma_{X}$. If $W_{i} \in K_{\Sigma_{X}}$, then $B N_{\gamma, \beta}\left(Y_{i}\right)=\beta e_{B}^{T}$, which means that the sphere collapses to a mean vector. In this case, the generalization of network will decrease as studied in (Morcos et al. 2018).

- If batch size $B \ll n$, the dimension of kernel $\operatorname{dim}\left(K_{\Sigma_{X}}\right) \geq n-B$ will be large. That is to say $W_{i}$ will lie in or very close to $K_{\Sigma_{X}}$ with very high probability. For example, in the extreme case where $B=2$, $B N\left(Y_{i}\right)=(-1,1)$ or $B N\left(Y_{i}\right)=(1,-1)$.

- If batch size $B \geq n$, this setting may avoid the above situation to some extent, but in real application, we cannot have a very big batch size due to the memory constraint. Also large batch would decrease generalization as studied in (Luo et al. 2019). Thus, choosing an appropriate batch size is an important question.

Additionally, from Eq. (11), we can see that BN puts constraint on row vectors of weight matrix $W$, namely

$$
\begin{aligned}
B N_{\gamma, \beta}\left(Y_{i}\right) & =\frac{\gamma W_{i}}{\sqrt{W_{i} \Sigma_{X} W_{i}^{T}}}(X-\bar{X})+\beta e_{B}^{T} \\
& =W_{i}^{\prime} X+b^{\prime}
\end{aligned}
$$

where $W_{i}^{\prime}=\frac{\gamma W_{i}}{\sqrt{W_{i} \Sigma_{X} W_{i}^{T}}}$, and $b^{\prime}=\beta e_{B}^{T}-\frac{\gamma W_{i} \bar{X}}{\sqrt{W_{i} \Sigma_{X} W_{i}^{T}}}$. Therefore, $\mathrm{BN}$ is scaling invariant for both weights $W$ and inputs $X$, also invariant with bias with row vectors of $X$. We can see that $W_{i}^{\prime}$ lies on a $\operatorname{rank}(\Sigma)$ dimension ellipsoid (Cho and Lee 2017) (Kohler et al. 2019), and the ellipsoid converges as the training converges. Thus, batch normalization constrains our effective weight $W_{i}^{\prime}$ on an ellipsoid which is a compact set. We should notice that the ellipsoid is changing over training steps, which means the units adjust the effective weights to achieve better performance.

\subsection{Layer Normalization}

In this section, we make a deep analysis of layer normalization based on Lemma 1. Specifically, layer normalization (LN) (Ba, Kiros, and Hinton 2016) normalizes inside a layer, whose input is $X=\left(X_{1}, X_{2}, \ldots, X_{n}\right)^{T} \in \mathbb{R}^{n}$, and the weight is $W \in \mathbb{R}^{m \times n}$. Thus $Y=\left(Y_{1}, Y_{2}, \ldots, Y_{m}\right) \in \mathbb{R}^{m}$. Let $e_{m}=(1,1, \ldots, 1)^{T} \in \mathbb{R}^{m}$, then the mean of $Y$ is $\bar{Y}=\frac{1}{m} e_{m} \sum_{i=1}^{m} Y_{i}=\frac{1}{m} e_{m} e_{m}^{T} Y$. Layer normalization for $Y$ is

$$
\begin{aligned}
L N_{\gamma, \beta}(Y) & =\gamma \sqrt{m} \frac{Y-\bar{Y}}{\|Y-\bar{Y}\|_{2}}+\beta e_{m} \\
& =\gamma \sqrt{m} \frac{(W-\bar{W}) X}{\|(W-\bar{W}) X\|_{2}}+\beta e_{m}
\end{aligned}
$$


where $\bar{W}=\frac{1}{m} e_{m} e_{m}^{T} W . \mathrm{LN}$ is scaling invariant for both weights $W$ and inputs $X$, and is moving invariant with row vectors of $W$. Every row of $\bar{W}$ is

$$
\bar{W}_{i}=\left(\frac{1}{m} \sum_{k=1}^{m} W_{k}^{1}, \frac{1}{m} \sum_{k=1}^{m} W_{k}^{2}, \ldots, \frac{1}{m} \sum_{k=1}^{m} W_{k}^{n}\right) \in \mathbb{R}^{n}
$$

for $i=1, \ldots, m$.

Thus $\bar{W}_{i}$ is the mean of all the row vectors in $W$. It is easily observed from above Eq. (13) that LN is equivalent to centering the row vectors of $W$ and then normalizing ( $W-$ $\bar{W}) X$ on $\mathbb{S}_{\sqrt{m}}^{m-2}$ (more details can be found in Appendix).

\subsection{Weight-based Normalization Methods}

In this section, we make a deep analysis of weight-based normalization methods. This kind of normalization constrains the effective weights on a sphere rather than ellipsoid as data-based normalization does. In this way, data-based normalization is much flexible to fit data and obtain good performance. It seems that both $\mathrm{BN}$ and $\mathrm{LN}$ do normalization to data, but they implicitly do transformation of weights as characterized above. IN and GN follow the same way as LN, so we do not present the deduction here. First, weight normalization (WN) (Salimans and Kingma 2016) is a direct way to normalize weight rather than inputs. It splits the weights' row vector as length and direction like BN, and updates both of them in the training. WN takes the form as

$$
W_{i}=g \frac{V_{i}}{\left\|V_{i}\right\|_{2}}
$$

where $g$ is the length of $W_{i}$ and $V_{i}$ is the direction of the row vector. We can see that if $\Sigma_{X}=I d$, then BN degenerates to WN. That is, if the covariant matrix is identity or the vectors of the data matrix in every layer are independent, then $\mathrm{BN}$ will degenerate to WN. Of course, it is almost impossible to make all the layers independent in $\mathrm{BN}$. BN performs better and also widely used in many tasks. One of the possible reasons is that it considers the relationship between different samples which shows as the direction of the vector in Fig 2.

Spectral normalization (SN) (Miyato et al. 2018) was proposed to train discriminator of GAN and has obtained better stability. The idea is to constrain the Lipschitz constant of the network by normalizing weight. Precisely,

$$
W_{S N}(W)=\frac{W}{\sigma(W)}
$$

where $\sigma(W)=\max _{\|h\|_{2}=1}\|W h\|_{2}$ is the spectral norm of $W$, namely the biggest singular value of $W$.

Centered weight normalization (CWN) (Huang et al. 2017) and weight standardization (WS) (Qiao et al. 2019) both normalize weights' row vector by centering and scaling,

$$
\begin{aligned}
W S\left(W_{i}\right) & =\frac{W_{i}-\bar{W}_{i}}{\sigma_{W_{i}}}=\sqrt{n} \frac{W_{i}-\bar{W}_{i}}{\left\|W_{i}-\bar{W}_{i}\right\|_{2}} \\
& =\sqrt{n} C W N\left(W_{i}\right)
\end{aligned}
$$

where $\bar{W}_{i}=\frac{1}{n} W_{i} e_{n} e_{n}^{T}$ is mean of row vector $W_{i}$, and $e_{n}=(1,1, \ldots, 1)^{T} \in \mathbb{R}^{n}$. WS is equivalent to CWN which only normalizes by $L_{2}$ norm of a centered row vector. WS puts the row vector of weight on a sphere $\mathbb{S}_{\sqrt{n}}^{n-2}$, while CWN moves row of weight on unit sphere $\mathbb{S}_{1}^{n-2}$.

\subsection{Symmetry of Normalization}

Most of the aforementioned normalization methods are scaling invariant, which will lead us to do optimization on the space by dividing the scaling symmetry (Cho and Lee 2017; Meng et al. 2019). To be more precise, the scaling invariant property allows us to pay more attention on the direction of weights instead of the magnitude. No matter how big or small the weights are, the effective weights don't change much. Here effective weights mean the weights multiplied by $X$, like $W_{i}^{\prime}$ in Eq. (12). This stables the training process and helps to obtain better performance than those without normalization.

We need another lemma from the symmetric group view to give us useful intuitions about normalization. In classical mechanics, symmetry is always related to invariant and is identified as Nother's Theorem (Arnol'd 2013). In our case, because the weights are scaling invariant for many normalization methods, we just study the identity related to scaling group. It tells us that the weight is always orthogonal to its gradient, thus the norm of weights will keep increasing with stochastic gradient descent (SGD).

Lemma 2 If $f(x)$ is a scaling invariant differentiable function on $\mathbb{R}^{n}$, i.e. $f(\lambda x)=f(x), \forall \lambda \in \mathbb{R}$, then

$$
x^{T} \nabla f(x) \equiv 0 .
$$

where $\nabla f(x)$ is the gradient at $x$. This implies that $x$ is always orthogonal to its gradient, $x \perp \nabla f(x)$.

Proof 2 As $f(\lambda x)=f(x)$, differentiating with respect to $\lambda$ and evaluating at $\lambda=1$, we have

$$
\begin{aligned}
0 & \left.\equiv \frac{d}{d \lambda}\right|_{\lambda=1} f(\lambda x)=\frac{d f(\lambda x)}{d(\lambda x)} \frac{d(\lambda x)}{d \lambda} \\
& =x^{T} \frac{d f}{d x}(x)=x^{T} \nabla f(x) .
\end{aligned}
$$

Thus the proof is completed.

With the above setting, we just give an analysis on a neuron $i$ of one layer for simplicity, and the analysis applies to all the weights. Let the loss function be $\mathcal{L}(W)$. Since $\mathcal{L}(W)$ is scaling invariant for every row $W_{i}$ of $W$, i.e. $\mathcal{L}(\Lambda W)=\mathcal{L}(W)$, and for any diagonal matrix $\Lambda=$ $\operatorname{diag}\left(\lambda_{1}, \lambda_{2}, \ldots, \lambda_{m}\right) \in \mathbb{R}^{m \times m}$. By Lemma 2 , we directly have

Lemma 3 If loss function $\mathcal{L}(W)$ is scaling invariant with every row $W_{i}$ of $W$, then

$$
W_{i} \perp \nabla_{W_{i}} \mathcal{L}(W) .
$$

Thus, for the SGD process, $W_{i}^{k+1}=W_{i}^{k}-\eta \nabla_{W_{i}} \mathcal{L}\left(W^{k}\right)$, the norm of $W_{i}^{k+1}$

$$
\left\|W_{i}^{k+1}\right\|_{2}^{2}=\left\|W_{i}^{k}\right\|_{2}^{2}+\eta^{2}\left\|\nabla_{W_{i}} \mathcal{L}(W)\right\|_{2}^{2} .
$$

This implies that the norm of weights will keep increasing. 

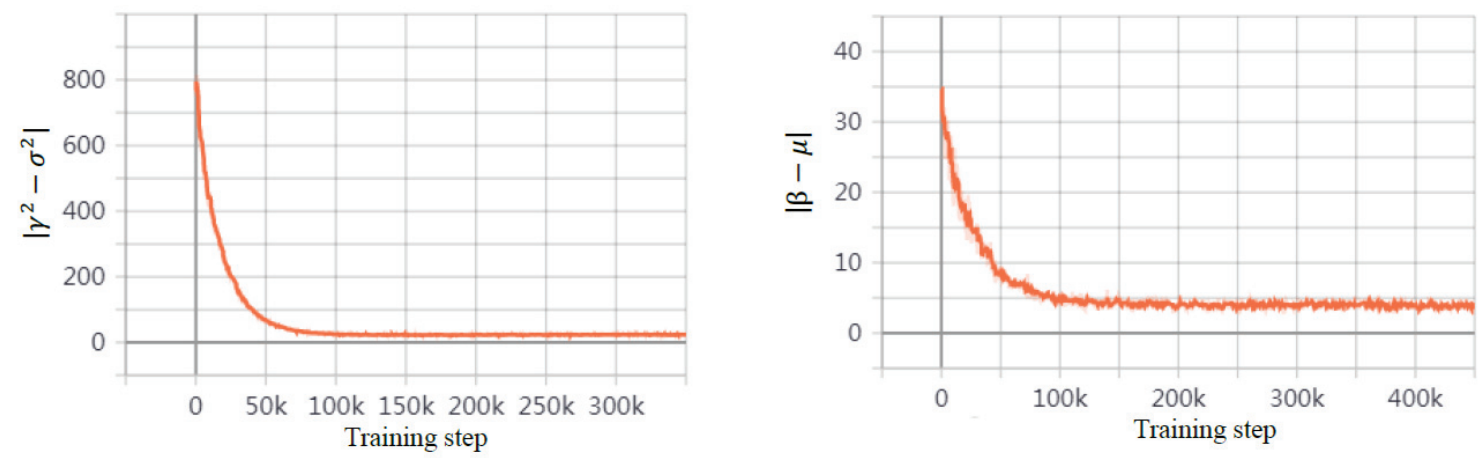

Figure 2: The change of $\left|\gamma^{2}-\sigma^{2}\right|$ and $|\beta-\mu|$ in training process.

\begin{tabular}{|c|c|c|c|c|c|c|c|c|}
\hline Model & BN & BN+WD & GN & GN+WD & LN & LN+WD & WN & WN+WD \\
\hline Clean & 0.9687 & 0.9375 & 0.9687 & 0.9375 & 0.8711 & 0.9687 & 0.8679 & 0.9062 \\
Gaussian Noise & 0.8125 & 0.7812 & 0.9062 & 0.8437 & 0.8437 & 0.8002 & 0.7060 & 0.7187 \\
Acc-Diff1 & 0.1562 & 0.1563 & 0.0625 & 0.0938 & 0.0274 & 0.1685 & 0.1619 & 0.1875 \\
BIM- $l_{\infty}$ & 0.5380 & 0.6000 & 0.5793 & 0.5815 & 0.5253 & 0.5333 & 0.5031 & 0.4687 \\
Acc-Diff2 & 0.4307 & 0.3375 & 0.3894 & 0.3560 & 0.3458 & 0.4354 & 0.3648 & 0.4375 \\
\hline
\end{tabular}

Table 1: Test accuracy of ResNet101 on CIFAR-10

All the aforementioned normalization methods, including $\mathrm{BN}, \mathrm{LN}, \mathrm{IN}, \mathrm{GN}, \mathrm{WN}, \mathrm{CWN}$, and $\mathrm{WS}$, are scaling invariant. Therefore, training with these normalization methods will keep weights increasing. This was first observed in (Salimans and Kingma 2016) for WN. Here we make a further step and obtain the same result for all the scaling invariant normalization methods, which is also found in (Arora, $\mathrm{Li}$, and Lyu 2018).

However, the increasing weight may cause adversarial vulnerability as it will amplify the attack. As mentioned in (Galloway et al. 2019), batch normalization is a cause of adversarial vulnerability. We conclude that the adversarial vulnerability exists in all the scaling invariant normalization methods, not limited to batch normalization, and weight decay can reduce the vulnerability to some extent (Hoffer et al. 2018; Zhang et al. 2018). In the experimental section, we conduct experiments to verify this point.

Though it is easy to get Lemma 3 from Lemma 2, we also prove it for batch normalization with very careful computation as (Santurkar et al. 2018)(see details in Appendix 1.5). Also, it is obvious that we can get the term $\left\langle\nabla_{\mathbf{y}_{j}} \mathcal{L}, \hat{\mathbf{y}}_{j}\right\rangle$ vanishing in (Santurkar et al. 2018) with BN by Lemma 2.

\section{Experiments}

In this section, we conduct a series of experiments to verify the claims of normalization methods induced by our proposed analysis tools. Specifically, we mainly focus on batch normalization (BN) method and adversarial vulnerability for scaling invariant normalization. The experiments are conducted on CIFAR-10 or CIFAR-100 dataset where images are normalized to zero mean and unit variance. Additionally, the training samples are also augmented by left-right flipping. Since ResNet (He et al. 2016) has been verified to achieve the state-of-the-art performance in the image classification task, we thus use the ResNet-101 as our baseline network in the experiment.

\subsection{Convergence of Parameters for $\mathrm{BN}$}

Firstly, we conduct experiments to verify the claim that $\gamma^{2}$ is the approximation of variance $\sigma^{2}$ for units' pre-activation vector, and $\beta$ is the approximation of mean $\mu$ as shown in Section 2.2. In other words, the claim indicates that the value of $\left|\gamma^{2}-\sigma^{2}\right|$ and $|\beta-\mu|$ should stabilize at a small value when the training converges.

Specifically, in this experiment, we train ResNet-101 model on CIFAR-10 using the SGD algorithm with learning rate $10^{-3}$ and epoch number 200. Focusing on the first BN module in the first residual block, we show the change of $\left|\gamma^{2}-\sigma^{2}\right|$ and $|\beta-\mu|$ as iteration goes in Fig 2. There are 64 feature layers for BN module and each layer has its own $\gamma_{i}, \sigma_{i}, \beta_{i}, \mu_{i}$. Since they have similar changing patterns, we randomly choose one pair of values to show in Fig 2. From Fig. 2, it can be easily seen that the parameters $\gamma$ and $\beta$ are of great importance in the training process. The convergence of $\gamma$ and $\beta$ is related to the convergence of training neural networks. The distance between $\gamma$ and $\sigma$ keeps stable at a small value after the model converges, so does the distance between $\beta$ and $\mu$.

\subsection{Adversarial vulnerability for scaling invariant normalizations}

In this section, we show the relationship between adversarial vulnerability and scaling invariant normalization methods. As we claim in Lemma 3, the norm of weights keeps increasing in the training process for scaling invariant normalization methods. We conduct experiments on CIFAR-10 


\begin{tabular}{|c|c|c|c|c|c|c|c|c|}
\hline Model & BN & BN+WD & GN & GN+WD & LN & LN+WD & WN & WN+WD \\
\hline Clean & 0.6875 & 0.6563 & 0.6257 & 0.6063 & 0.6358 & 0.6279 & 0.6147 & 0.6072 \\
Gaussian Noise & 0.4596 & 0.4953 & 0.4098 & 4327 & 0.3983 & 0.4278 & 0.3476 & 0.3874 \\
Acc-Diff1 & 0.2279 & 0.1610 & 0.2159 & 0.1736 & 0.2375 & 0.2001 & 0.2671 & 0.2198 \\
BIM- $l_{\infty}$ & 0.1397 & 0.1558 & 0.2366 & 0.2813 & 0.2354 & 0.2531 & 0.1647 & 0.1965 \\
Acc-Diff2 & 0.5478 & 0.5005 & 0.3891 & 0.3250 & 0.4004 & 0.3748 & 0.4500 & 0.4077 \\
\hline
\end{tabular}

Table 2: Test accuracy of ResNet-101 on CIFAR-100
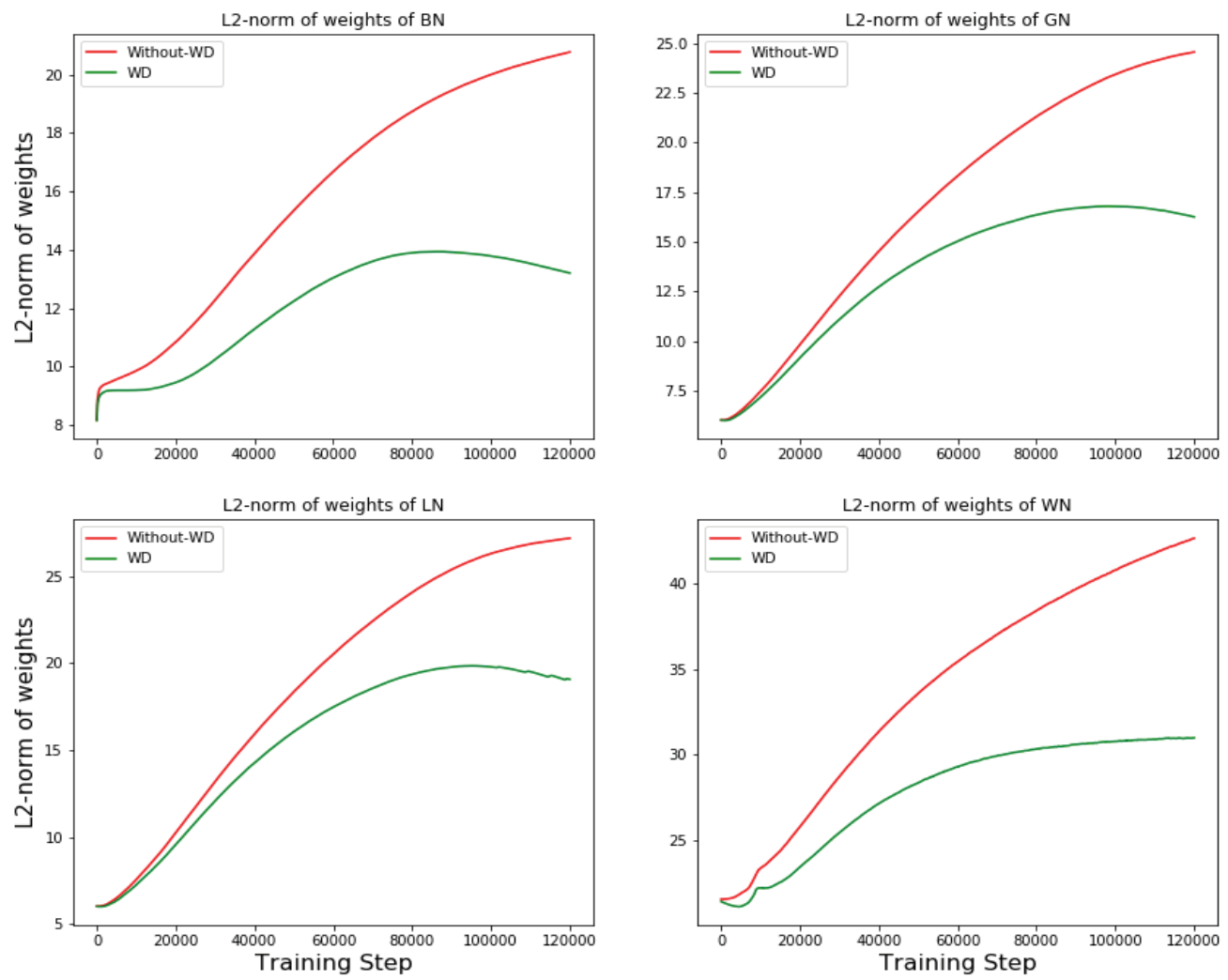

Figure 3: L2-norm of weights of different normalization methods (with or without WD) in training process.

and CIFAR-100 datasets using the ResNet-101 model with learning rate $10^{-3}$ and epoch number 200 .

Focusing on the first batch normalization module in the first residual block, we show the $L_{2}$-norm of weights of convolutional layers. The $L_{2}$-norm curves with $\mathrm{BN}, \mathrm{LN}, \mathrm{GN}$ and WN are shown in Fig. 3. Additionally, we evaluate the robustness of convolutional networks with and without the weight decay strategy, which are trained under the same settings as before. Considering the simplicity and ability to degrade performance with little perceptible change to the input, we apply white-box adversarial attack-BIM and additive Gaussian noise to original clean data. The setting details are the same as (Galloway et al. 2019). The test accuracy of the same model structure with different normalization methods are shown in Table 2, where "+WD" denotes whether weight decaying is added.

The weight norms of all the normalization methods we consider are increasing as training goes. By further adding the weight decay, the weight norm stabilizes early or even has a minor decrease. Also, from Table 2 we can see that accuracy decreases with noise and BIM attacking in the same pattern. This implies that all these scaling invariant normalization methods have the same problem of adversarial vulnerability. One of the reasons for this problem is that increasing weight magnitude can amplify the noise or attack in the deep network. Additionally, we find that with weight decay the accuracy increases a bit than that without weight decay. That is because weight decay breaks the scaling invariant property so that the weight norm stabilizes early or even decreases, as shown in Fig. 3.

\section{Conclusion and Future work}

This paper makes a deep analysis on most of the normalization methods according to the unified mathematical analysis tool and then puts forward some useful claims and conclusions. More specifically, firstly, most normalization methods 
can be interpreted as normalizing pre-activations or weights onto a sphere. Secondly, a new network pruning strategy based on centering parameter and scaling parameter of $\mathrm{BN}$ is proposed, and that the batch size of $\mathrm{BN}$ should be set close to the width of the network is also claimed. We will explore these in our future works. Thirdly, optimization on module space which is scaling invariant can help to stabilize the training of the network. Fourthly, we show that training with these normalization methods keeps weights increasing, which aggravates adversarial vulnerability since it will amplify the attack. A series of experiments have been conducted to verify the claims and conclusions. Much work still needs to be done to explore this topic in the future.

Acknowledgments The second author was supported by the China Postdoctoral Science Foundation funded project (2018M643655), the Fundamental Research Funds for the Central Universities, and the China NSFC project under contract 61906151.

\section{References}

Arnol'd, V. I. 2013. Mathematical methods of classical mechanics, volume 60. Springer Science \& Business Media.

Arora, S.; Li, Z.; and Lyu, K. 2018. Theoretical analysis of auto rate-tuning by batch normalization. arXiv preprint arXiv:1812.03981.

Ba, J. L.; Kiros, J. R.; and Hinton, G. E. 2016. Layer normalization. arXiv preprint arXiv:1607.06450.

Bjorck, N.; Gomes, C. P.; Selman, B.; and Weinberger, K. Q. 2018. Understanding batch normalization. In Advances in Neural Information Processing Systems, 7694-7705.

Cho, M., and Lee, J. 2017. Riemannian approach to batch normalization. In Advances in Neural Information Processing Systems, 5225-5235.

Galloway, A.; Golubeva, A.; Tanay, T.; Moussa, M.; and Taylor, G. W. 2019. Batch normalization is a cause of adversarial vulnerability. arXiv preprint arXiv:1905.02161.

He, K.; Zhang, X.; Ren, S.; and Sun, J. 2016. Deep residual learning for image recognition. In Proceedings of the IEEE conference on computer vision and pattern recognition, 770-778.

Hoffer, E.; Banner, R.; Golan, I.; and Soudry, D. 2018. Norm matters: efficient and accurate normalization schemes in deep networks. In Advances in Neural Information Processing Systems, 2160-2170.

Huang, L.; Liu, X.; Liu, Y.; Lang, B.; and Tao, D. 2017. Centered weight normalization in accelerating training of deep neural networks. In Proceedings of the IEEE International Conference on Computer Vision, 2803-2811.

Ioffe, S., and Szegedy, C. 2015. Batch normalization: Accelerating deep network training by reducing internal covariate shift. arXiv preprint arXiv:1502.03167.

Kohler, J.; Daneshmand, H.; Lucchi, A.; Hofmann, T.; Zhou, M.; and Neymeyr, K. 2019. Exponential convergence rates for batch normalization: The power of length-direction decoupling in non-convex optimization. In The 22nd Inter- national Conference on Artificial Intelligence and Statistics, 806-815.

Liu, Z.; Li, J.; Shen, Z.; Huang, G.; Yan, S.; and Zhang, C. 2017. Learning efficient convolutional networks through network slimming. In Proceedings of the IEEE International Conference on Computer Vision, 2736-2744.

Luo, P.; Wang, X.; Shao, W.; and Peng, Z. 2019. Towards understanding regularization in batch normalization.

Masters, D., and Luschi, C. 2018. Revisiting small batch training for deep neural networks. arXiv preprint arXiv:1804.07612.

Meng, Q.; Zheng, S.; Zhang, H.; Chen, W.; Ma, Z.-M.; and Liu, T.-Y. 2019. G-sgd: Optimizing relu neural networks in its positively scale-invariant space. In ICLR 2019.

Mishkin, D., and Matas, J. 2015. All you need is a good init. arXiv preprint arXiv:1511.06422.

Miyato, T.; Kataoka, T.; Koyama, M.; and Yoshida, Y. 2018. Spectral normalization for generative adversarial networks.

Morcos, A. S.; Barrett, D. G.; Rabinowitz, N. C.; and Botvinick, M. 2018. On the importance of single directions for generalization.

Park, D. S.; Sohl-Dickstein, J.; Le, Q. V.; and Smith, S. L. 2019. The effect of network width on stochastic gradient descent and generalization: an empirical study. arXiv preprint arXiv:1905.03776.

Qiao, S.; Wang, H.; Liu, C.; Shen, W.; and Yuille, A. 2019. Weight standardization. arXiv preprint arXiv:1903.10520.

Salimans, T., and Kingma, D. P. 2016. Weight normalization: A simple reparameterization to accelerate training of deep neural networks. In Advances in Neural Information Processing Systems, 901-909.

Santurkar, S.; Tsipras, D.; Ilyas, A.; and Madry, A. 2018. How does batch normalization help optimization? In $\mathrm{Ad}$ vances in Neural Information Processing Systems, 24832493.

Ulyanov, D.; Vedaldi, A.; and Lempitsky, V. 2016. Instance normalization: The missing ingredient for fast stylization. arXiv preprint arXiv:1607.08022.

Wu, Y., and He, K. 2018. Group normalization. In Proceedings of the European Conference on Computer Vision (ECCV), 3-19.

Yang, G.; Pennington, J.; Rao, V.; Sohl-Dickstein, J.; and Schoenholz, S. S. 2019. A mean field theory of batch normalization. arXiv preprint arXiv:1902.08129.

Zhang, G.; Wang, C.; Xu, B.; and Grosse, R. 2018. Three mechanisms of weight decay regularization. arXiv preprint arXiv:1810.12281. 